\title{
Rate of ubiquitination and proteasomal degradation of topoisomerase I determines cellular resistance to cancer drug
}

Elizabeth C Unan ${ }^{1}$, Hiroya Matsuoka ${ }^{2}$, Hirofumi Hasuda ${ }^{2}$, Soumya Malhotra ${ }^{1}$, Siddhartha Pulukuri $^{1}$, Victor Bacelar ${ }^{1}$ Allison Berger ${ }^{3}$, Nibedita Chattopadhyay ${ }^{3}$, Lawrence Dick ${ }^{3}$, Eiji Oki ${ }^{2}$, Koji Ando ${ }^{1,2}$ and Ajit Bharti ${ }^{1 *}$

1. Department of Medicine, Division of Hematology Oncology, Boston University School of Medicine, 88 East Newton Street, Boston, MA 02118 USA

2. Department of Surgery and Science, Graduate School of Medical Sciences, Kyushu University, 3-1-1 Maidashi, Higashi-ku Fukuoka City, Fukuoka, Japan 8128582

3. Takeda Pharmaceutical Company Limited, Cambridge, MA 02139, USA

* To whom correspondence should be addressed 
Common Summary: Topotecan and irinotecan (camptothecin analogues, CPTs) specifically inhibit topoI and are used to treat various solid tumors. Third generation topoI inhibitors, such as Onivyde, and two antibody drug conjugates (ADCs), sacituzumab govitecan (Trodelvy) and trastuzumab deruxtecan (Enhertu), have shown higher patient response rates. Taken together, topoI inhibitors represent one of the most potent classes of anticancer drugs; however, the response rate in this class of drugs remains low, and drug resistance mechanisms are not well understood. Importantly, the rapid rate of CPT induced proteasomal degradation of topoI has been shown to be the primary mechanism of drug resistance. We have elucidated the CPT induced UPP mediated topoI degradation pathway, which provides unique opportunities to develop a predictive biomarker for this class of drugs and potentially identify drug targets for novel combination therapy to overcome CPT resistance. Here, we report that proteasome inhibitors stabilize CPT induced topoI degradation and reduce drug resistance.

Abstract: Targeted ubiquitination and proteasomal degradation regulates various cellular pathways, and the discovery that CPT induced rapid proteasomal degradation of topoI is the primary mechanism of CPT resistance was novel and compelling. CPTs are used extensively to treat various solid tumors like colorectal, gastric, pancreatic, ovarian and small cell lung cancer. The response rate is low and the classical mechanisms of drug resistance are yet to be validated. Remarkably, CPT resistant cells degrade topoI rapidly by UPP and recently we've published the molecular determinants of this pathway. To further understand the UPP mediated CPT resistance mechanism we used targeted proteasome prohibition by ixazomib (MLN9708) to stabilize topoI and determine its impact on CPT resistance. CPT resistant and sensitive cancer lines were both treated with ixazomib in combination with CPT. The CPT induced rate of topoI degradation, and its stabilization by ixazomib, was visualized and estimated by immunohistochemistry, immunofluorescence and immunoblotting assays, MTT and subG1 assays provided the drug sensitivity. Our results demonstrate that cells that degrade topoI rapidly are very resistant to CPT, and ixazomib significantly inhibits CPT induced topoI degradation. Notably, inhibition of 
proteasomal degradation by ixazomib overcomes the drug resistance and sensitizes the cells for CPTs. 


\section{Introduction:}

UPP pathway in different disease and drug resistance: The ubiquitin proteasome system (UPS) is integral for multiple cellular pathways and processes, some of which include cell cycle regulation, DNA repair, and protein degradation. Disruption or alteration of the ubiquitin proteasome pathway (UPP) during these processes often results in pathological disease. As notable examples, deregulation of UPS can lead to various cancers and neurodegenerative disorders. One of the main functions of the UPS is protein regulation and degradation, and when proteostasis is disrupted several neurodegenerative diseases may develop. Normal regulation for misfolded proteins is turnover by the UPP, however in these disease states this key protective mechanism is impaired [1].

A key feature of cancers is their ability to divide and grow rapidly despite accumulated mutations and DNA damage. The UPS in normal states acts to modulate proteins, of which p53 modulation through E3 ligase MDM2 is an important example. Activation of MDM2 results in p53 degradation, which removes an important inhibition on cell growth. Overexpression or activation of MDM2 is therefore associated with progression of tumor growth. However, ubiquitination processes are not limited to activation of an oncogenic pathway, but may also inhibit it based on the regulator acted upon [2]. The diverse nature of UPP targets allows this wide breadth of downstream effects if UPP is dysregulated. Focusing on one aspect of the UPP, E3 ligases offer specificity within the system because they act in substrate recognition and ubiquitin transfer to the targets [3]. Deregulated E3 ubiquitin ligases have been shown to be widely involved in the development of several cancers [4]. As previously discussed, MDM2 is an E3 ligase involved with p53 ubiquitin associated proteasomal degradation. An upregulation of MDM2 is associated with poor prognosis in cancer patients; in addition, an upregulation of the RING1 protein has been found to facilitate MDM2's ligase action and further support cell growth [2]. E3 ligases also act on tumor suppressor proteins. A prominent representation of this is the UPP ligase complex associated with von Hippel-Lindau tumors. Hypoxia inducible factor 
(HIF) is a well-researched target of the von Hippel Lindau protein (pVHL), which is a E3 ligase. HIF plays an indispensable role in the cellular response to hypoxia and resulting angiogenesis. When both copies of VHL are lost, HIFs are stabilized and allowed to promote an unregulated hypoxia response and angiogenesis, which increases tumor cell survival. Tumors associated with loss of VHL include clear cell renal cell carcinoma and pancreatic neuroendocrine tumors [4]. The widespread involvement of the UPS in various disease states warrants consideration of targeting the UPS pharmacologically. Proteasome inhibitors (PI) have been used in cancer treatment regimens for over 15 years and have had mixed success due to its lack of efficacy in solid tumors. Bortezomib, for the treatment of multiple myeloma and mantle cell lymphoma, is an important example as it is the first PI to be utilized in clinic [5]. Use of Bortezomib widened to some solid tumors, however the need for combination treatments arose due to lack of efficacy and potentially acquired resistance. Multiple modes of resistance are being considered, such as the induction of the IGF-1 pathway, mutations in PSMB5, and induction of heat shock proteins (HSPs) [5]. Thus, the drug resistance mechanisms of proteasome inhibitors are partly understood. Notably, the UPS plays important role in imparting drug resistance by degrading the drug targets, and here we will discuss one such pathway.

UPS Dependent Cancer Drug Resistance: Most of the drug sensitivity studies identified single gene expression or gene mutations for targeted therapies like the KRAS mutation and the HER2-IHC test; however, very little is known about mechanisms of resistance to chemotherapeutic agents. It's important to mention that other than breast and prostate, approximately $50 \%$ of all solid tumor patients are treated with chemotherapy alone, or in combination with targeted or radiation therapy (ACS report 2019-2021). Large-scale pharmacogenomics studies, including genome-wide association studies (GWAS), did not provide any definitive molecular signature for specific chemotherapeutic agents. The Cancer Cell Line Encyclopedia and the Genomics of Drug Sensitivity in Cancer (GDSC) used more than 600 cancer cell lines and 150 cancer drugs, including chemotherapeutic agents. Apart from the known predictors, AHR expression was associated with MEK inhibitor efficacy in NRAS-mutant lines and SLFN11 expression predicted sensitivity to topoI inhibitors $[6,7]$. Neither of these novel findings has been validated yet in 
clinical settings and SLFN11 expression is not specific only to topoI inhibitors. The rate of CPT induced topoI degradation by UPP is major determinant of the drug sensitivity. First, we'll review the proposed mechanisms of CPT resistance and then discuss the UPP mediated drug resistance. We will also provide data to support UPP mediated mechanism of resistance and potential use of proteasome inhibitor to overcome the resistance.

Mechanisms of CPT resistance: Irinotecan specifically targets topoI and is in clinical use as first line therapy for metastatic colorectal cancer (mCRC) and pancreatic cancer and $2^{\text {nd }}$ line therapy for gastric cancer [8-12]. Another CPT analogue, topotecan is used in second line therapy to treat ovarian and small cell lung cancer (SCLC) patients. Notably, recent approval of two antibody drug conjugates (ADCs), Trodelvi (Scituzumab govitecan) and Enhertu (trastuzumab deruxtecan) with topoI inhibitors as the cytotoxic warheads, has placed this class of drug front and center in future anticancer drug discovery efforts. However, only $13 \%-32 \%$ of patients respond to these drugs and the mechanism of resistance is not understood [13-21]. Activation of $\mathrm{ABC}$ transporters/multi-drug resistance (MDR) genes, mutations in topoI and CPT induced rapid degradation of topoI by UPP were three proposed CPT resistance mechanisms.

(i) ABC transporters/MDR gene mediated mechanisms: Several pharmacogenomics studies using NCI-60 cell lines to determine drug response and gene expression profiles were conducted. The studies included several topoI inhibitors, but prediction of drug response based on gene expression profiles was very limited. Notably, no relationship was observed between MDR/ABC transporter gene expression and CPT response. These findings were later confirmed where 14 human $\mathrm{ABC}$ transporter genes were studied and none showed a relationship to CPT response [22]. A more comprehensive genomics study of all known ABC transporter genes in the NCI-60 cell lines confirmed that CPTs are not substrate for ABC transporter genes [23].

(ii) TopoI gene mutations and CPT resistance: There are 13 topol gene point mutations reported (Fig.1), representing both acquired by conditional drug resistance development [24-29] and intrinsic gene mutations in patients and CPT producing plant species [30-33]. 
A closer analysis of the sites of these mutations and topol functional domain organization indicates that they are either localized in the DNA binding domain (amino acids 361, 363, $370,418,533,583$ ) or the catalytic region (amino acids 722 and 729). Both domains are highly conserved and play critical roles in topoI enzyme activity. It is also important to note that amino acid 722 is the only one that is mutated in intrinsic and acquired CPT resistance. The other two mutations (amino acids 421 and 530) found in intrinsically resistant plant species are in close proximity to the mutations reported in acquired mutations (Fig. 1) [34]. A crystal structure of N722S clearly indicates the importance of this amino acid for nicking and re-ligation reaction to reduce supercoiling of DNA by topoI [28]. Other reported mutations, both intrinsic and acquired, provide a functional basis of CPT resistance as they either affect DNA-topoI binding or catalytic activity of the enzyme. However, the lack of intrinsic or acquired topoI mutations in patients indicate that topoI gene mutations don't account for CPT resistance. (30).
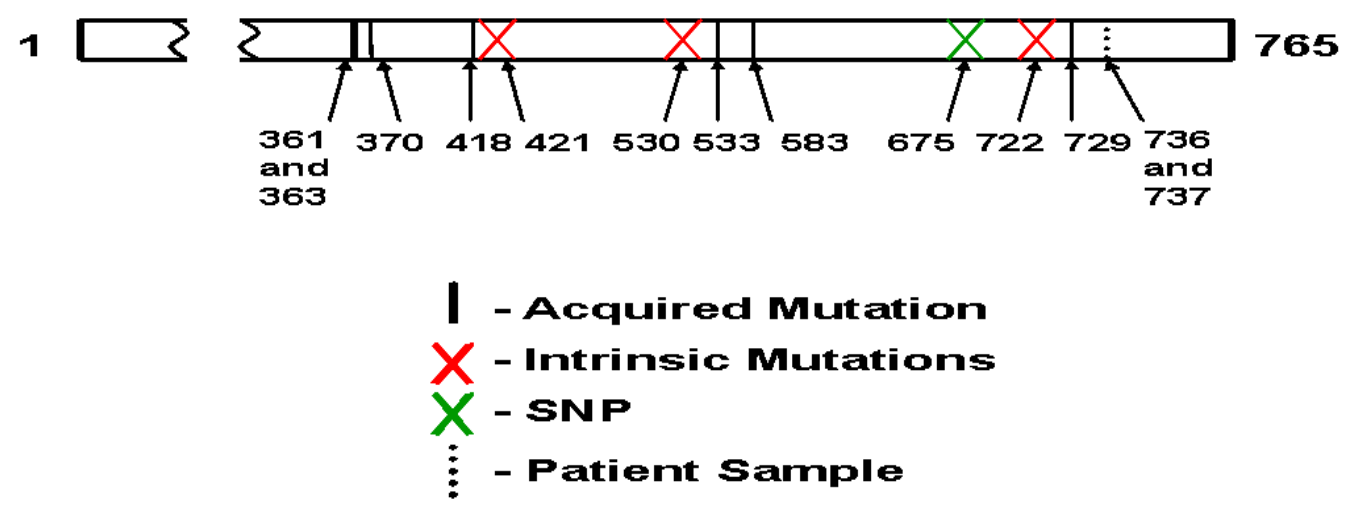

Figure 1 : A Schematic Representation of Known Topol Gene Point Mutations

(iii) Rate of UPP dependent degradation of topoI in the response to CPT: One of the most distinct cellular responses to CPT is the degradation of topoI by the UPP. It was first observed in peripheral leukocytes of patients receiving 9-amino camptothecin [35], and subsequently in multiple cancer cell lines [36]. More importantly, Desai et al. demonstrated that the irinotecaninduced degradation of topoI is mediated by ubiquitin proteasomal pathway, cells degrade topoI 
differentially, and the cells that degrade topoI rapidly are resistant to irinotecan $[37,38]$. To understand the CPT induced proteasomal degradation of topoI, Ando et al. used a functional proteomics approach to isolate and identify topoI interacting protein complex and have published that: i) topoI associates with DNA-PK, and DNA-PKcs phosphorylates topoI at Serine 10; ii) Phosphorylated topoI is ubiquitinated by BRCA1; iii) cells with higher basal levels of topoIpS10 degrade topoI rapidly and are resistant to irinotecan; iv) the higher basal level of topoIpS10 is maintained by phosphatase dependent activation of DNA-PKcs; v) nuclear phosphatase siRNA library screen identified PTEN and CTDSP1 enhances irinotecan induced topoI degradation and vi) silencing of PTEN enhanced DNA-PKcs activity and irinotecan resistance. Thus CPT induced topoI degradation pathway is a highly orchestrated interplay of posttranslational modifications of protein that determines the drug sensitivity [39].

\section{a) Higher basal level of phosphorylated topoI-pS10 is a molecular determinant of CPT} response: Our lab validated UPP mediated topoI degradation pathway at multiple levels. First, to determine if the resistant cells have higher levels of topoI-S10 phosphorylation, a phosphospecific topoI-S10 antibody was developed, characterized, and analytical validation of IHC assay was performed. The IHC assay, on several cell lines, indicated higher basal levels of topoI-pS10 in resistant cells [40]. We asked if this was true in a population of cancer patients. A multi cohort retrospective clinical validation study was conducted and the result demonstrated a strong relationship between higher topoI-pS10 level and CPT response. The study included eight cohorts of colorectal cancer $(n=176)$ patients, and two cohorts of gastric cancer $(n=106)$ FFPE slides from the patients treated with irinotecan as a single agent or in combination therapy. AntitopoI-pS10 immunohistochemically-stained slides were scanned and percent positive nuclei were quantitatively analyzed. Using a receiver operating characteristic (ROC) curve analysis, we determined that a threshold of $35 \%$ of topoI-pS10 positive nuclei would be a useful threshold for responder and non-responder patient populations. Using this threshold, we've demonstrated a 
very high positive and negative predictive value, both in the CRC and GC patient population [41].

b) BRCA1 ubiquitinates topoI: BRCA1 plays an important role in genome maintenance. Critical to many functions is its association with various protein complexes involved in DSB repair [42, 43]. However, the precise role of BRCA1 in DNA damage response (DDR) is not understood. In an effort to understand BRCA1 E3 ligase function in DNA-DSB, Reid et al. generated isogenic clones of embryonic stem cells with BRCA1-WT, I26A (a mutant that only impairs BRCA1's E3 ligase function and not BRCA1-BARD1 interaction), and a BRCA1 deletion mutant that affects BRCA1 DDR function significantly. Using mitomycin C (MMC) and ionizing radiation (IR), they demonstrated that E3 ligase deficient cells are not sensitized to these agents, and that the rates of homology directed DNA-DSB repair are similar [44]. In striking contrast, BRCA1-E3 ligase inactivation impairs the DDR when CPT is used as the DNA damaging agent in DT40 chicken cells. In these cells, V26A abrogates the E3 ligase activity of BRCA1. Cell viability, Rad51 foci formation and sister chromatid exchange (SEC) rates were all similar in BRCA1 WT and V26A cells in response to MMC. However, V26A mutant cells were very sensitive to CPT, with a sharp reduction in RAD51 foci formation and SEC frequency [45]. Collectively these studies demonstrate that BRCA1 E3 ligase function is critical only for CPT induced DDR. In our studies, we used three different approaches (highly purified in vitro ubiquitination assays, silencing of BRCA1, and expressing BRCA1-WT in HCC-1937, cells with BRCA1-deletion mutations) to demonstrate that BRCA1 ubiquitinates topoI in vitro and in cells. Our findings not only support the observations of Sato et al., but also define the mechanism of CPT sensitivity in BRCA1-E3 ligase deficient cells [39].

iv) Proteasome inhibitor reduces CPT induced topoI degradation: Work in our lab has clearly demonstrated that the cells that degrades topoI rapidly are more resistant to CPT. To validate this further, we asked if proteasome inhibitor would stall topoI degradation and 
overcome CPT resistance. First, we demonstrate more new data on how rate of proteasomal degradation of topoI is important in imparting CPT resistance, and then discuss how proteasomal inhibition interrupts topoI degradation and diminishes CPT resistance with new data.

a) The cells that degrade topoI rapidly are resistant to CPT: Studies of NCI 60 cell lines demonstrate wide-range of CPT drug sensitivity [39, 46]. We selected HCT15 (CPT resistant) and HCT116 (CPT sensitive) cells and treated them with SN38 (irinotecan metabolite) and determined the rate of topoI degradation by immunoblot analysis with anti-topoI. The data clearly demonstrated that, when treated with SN38, almost all topoI protein was degraded in HCT15 cells while in HCT 116 a minimal degradation of topoI protein was observed (Fig.2A). No topoI degradation was observed in cells treated with DMSO as control. A quantitative analysis of immunoblot from three independent experiments demonstrated $80-90 \%$ reduction in the topoI protein level when HCT 15 cells were treated with SN38. While in HCT 116 cells the topoI protein level was reduced only by $20-30 \%$ (Fig. 2B). To further validate these findings, we used mice xenograft models of CPT resistant (HCT15) and CPT sensitive (Colo205) cells. Mice were treated with CRLX101 (nano-linked CPT) and tumor tissue was harvested at 72 hours and 168 hours. The tumor tissue was fixed in formalin, embedded in paraffin, and FFPE slides were immunohistochemically (IHC) stained with anti-topoI. After 72 hours post treatement, minimal or no loss of topoI protein was observed in Colo205, while a complete loss of topoI was observed in HCT15 cell tumor xenograft. The immunohistochemical staining at 168 hours demonstrated two important findings. First, distinct loss of cell morphology in colo205 cells only, indicating potential cell death. Second, topoI protein level in HCT15 reappeared and was detectable by IHC with anti-topoI. Also no loss of cellular morphology was observed in the HCT15 cell tumor xenograft (Fig. 2C). To determine the drug efficacy, mice were treated with single dose of CRLX101 and tumor volume was measured up to $24^{\text {th }}$ day post treatment. The data indicates sharp reduction in Colo205 xenograft tumor volume after 18 days while no appreciable change was observed in HCT15 xenograft tumor (Fig. 2D). To demonstrate the 
apoptosis mediated cell death, FFPE slides from xenograft, were also immunostained with anticleaved caspase-3 (CC-3). Higher levels of DAB positive staining indicated a large percent of cells undergoing apoptosis only in Colo205 (Fig. 2E left panel) and not in HCT15 (Fig. 2E right panel) cell xenograft (Fig. 2E). Taken together, the rate of topoI degradation in HCT116 and Colo205 cells are slow and are sensitive to CPT. In contrast, HCT15 cells degrade topoI rapidly and are resistant to CPT.

A.

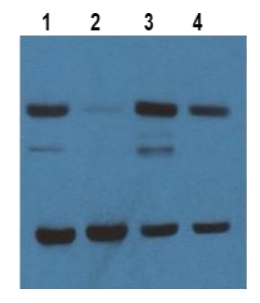

B.

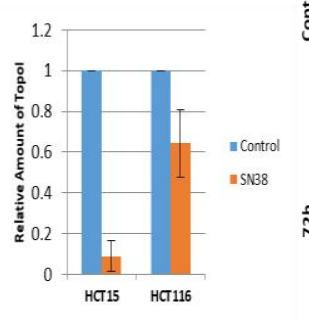

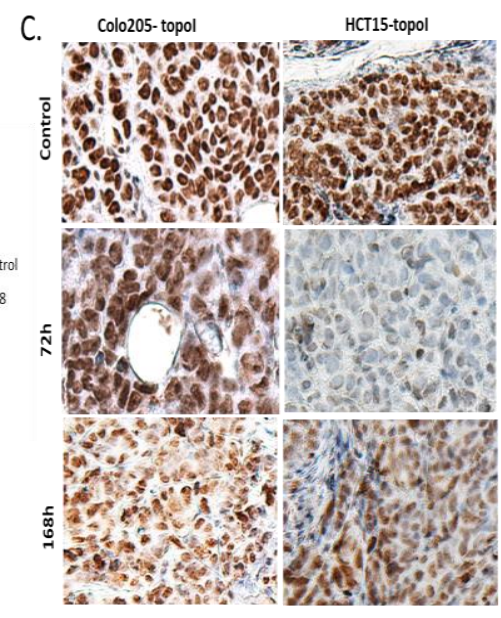

D.

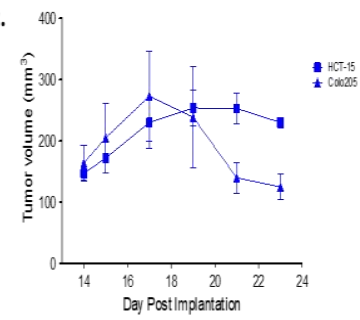

E.

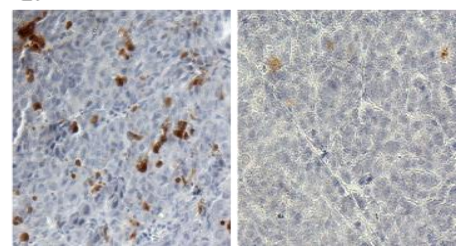

Figure 2. CPT induced rapid Topol degradation and drug resistance in cells and mice xenograft model. A. HCT15 and HCT116 cells were treated with either DMSO or SN38 $(2.5 \mu \mathrm{M})$ for three hours. Cells were harvested and cell lysates were analyzed by immunoblot analysis with anti-topol and anti- $\beta$-Actin. B. Three independent experiments were performed to determine CPT induced topol degradation (as in fig $1 \mathrm{~A}$ ) and the immunoblots were quantitatively analyzed to determine the relative amount of topol protein. $\mathbf{C}$. Mice with HCT 15 and Colo205 xenografts were treated with single dose of carrier polymer and CRLX-101, polymer linked- CPT, and tumors were harvested at 72 and 168 hours. FFPE slides from the tumor tissue were immunostained with anti-topol. D. Effect of single dose of CRLX101 $(10 \mathrm{mg} / \mathrm{kg})$ on tumor volume of HCT15 and Colo205 mice xenograft. E. FFPE slides from mice xenograft of Colo205 and HCT15, treated with CRLX101 and harvested at 72 hours, were immunostained with anti-cleaved caspase3 (CC3). Imaging of the immunostained slides were performed with Nikon bright field microscope.

\section{b) Proteasome inhibitor ixazomib stabilizes CPT induced topoI degradation: Genomically}

edited HCT15 cells (HCT15-473 as described in ref. 39) to express topoI-GFP fusion proteins were treated with SN38 or SN38 and ixazomib. After 3 hours cells were visualized using a confocal microscope to determine the change in topoI-GFP intensity. A significant reduction of fluorescence intensity was observed in cells treated with SN-38 after three hours compared to DMSO treated (control) cells (Fig. 3A left and middle panel). However, HCT15-473 cells pretreated with ixazomib showed minimal or no reduction in topoI-GFP intensity after SN-38 treatment (Fig. 3A right panel). Quantitative analysis of fluorescence intensity in three 
independent experiments clearly demonstrated that the SN-38 induced topoI-GFP degradation was significantly inhibited when the cells were pretreated with a proteasomal inhibitor (Fig. 3B). To further validate these findings, HCT15-473 cells were treated with DMSO, SN38, ixazomib, and ixazomib plus SN-38. After three hours, cells were harvested and cell lysates were analyzed by immunoblotting with anti-topoI. The results clearly demonstrated that SN38 induced topoI degradation was inhibited by ixazomib (Fig. 3C). We asked if the mechanism of topoI stabilization is similar in parental HCT15 cells. HCT15 cells were treated with SN38, ixazomib and the combination of SN38 and ixazomib. After 3 hours cells were analyzed by immunofluorescence analysis with anti-topoI. Significant reduction in topoI protein level was observed after 3 hours of SN38 treatment. Ixazomib treatment has no appreciable effect on topoI protein level. However, no change in topoI protein level was observed when cells were pretreated with ixazomib and then SN38 (Fig. 3D). The quantitative analysis of three independent immunofluorescence assay experiments demonstrates a significant CPT induced reduction in topoI protein level compared to both DMSO and ixazomib treated cells. Importantly, statistically significant stabilization of topoI was observed in cells pretreated with ixazomib and SN38 (Fig. 3E).

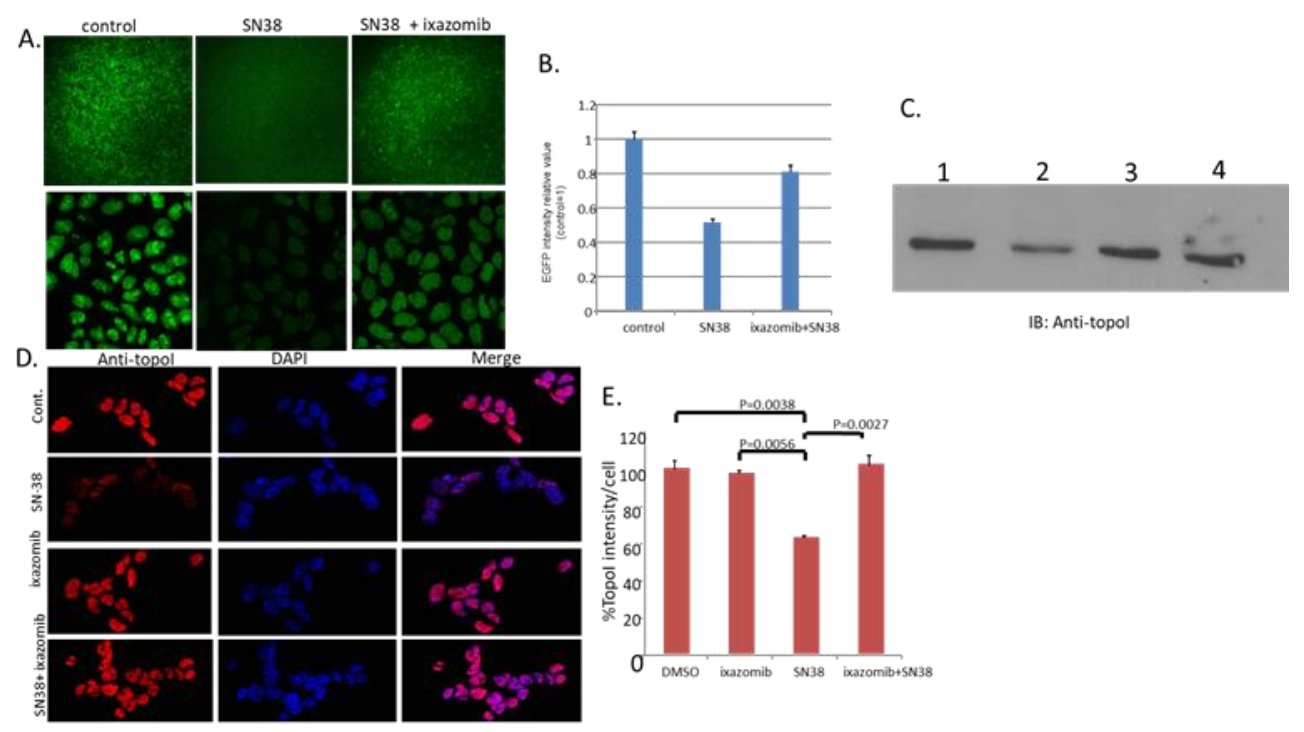




\section{Proteasome inhibitor mediated stabilization of topoI degradation enhances CPT sensitivity:}

HCT15 cells were treated with ixazomib (MLN-9708), SN38 and the combination of both drugs. To determine the effect of stabilization of SN-38 induced topoI degradation and its impact on drug sensitivity, cells were harvested at different time points after drug treatment and cell viability assays were performed. The cell viability assays clearly indicated a synergy in cell killing when cells were treated with SN38 and ixazomib compared to either ixazomib or SN38 alone (Fig. 3A). Using linear regression, $\mathrm{IC}_{50}$ was determined using data from three independent cell viability experiments. The results demonstrated that $\mathrm{IC}_{50}$ of SN38 and ixazomib alone was $1.5 \mu \mathrm{M}$ and $1.46 \mu \mathrm{M}$ respectively; however, when the drugs were combined the IC50 was reduced to $0.39 \mu \mathrm{M}$, indicating a significant reduction in drug resistance. To further confirm the drug combination synergy, HCT 15 cells were treated with SN38, ixazomib, and the combination of the two drugs, cell viability was determined by sub-G1 Analysis. In DMSO treated cells (control), irinotecan and ixazomib treated cells the \% sub-G1 population was 3.0, 5.28 and 8.36 respectively. However, when the two drugs were combined the $\%$ sub-G1 population, indicating apoptotic cells, increased to $22.3 \%$. Among the other cells, we identified HCC70, a triple negative breast cancer cell line, to be very resistant to CPTs. We asked if UPP mediated proteasomal degradation causes resistance in TNBC cells as well. Cell viability assays indicated the cells are very resistant to irinotecan; however, when ixazomib was added the cells were sensitized to irinotecan (Fig. 3D). It is important to mention that unlike HCT15, the TNBC cells are sensitive to ixazomib. However, at lower concentrations of ixazomib a significant synergy was observed when cells were treated with ixazomib and irinotecan. Using a different method, we determined the synergy of the drug combination in HCC70 cells. HCC70 cells were grown on matrigel and treated with SN38, ixazomib and the combination of both drugs. The bright field imaging was performed after 72 hours of the drug treatment, and this data indicates that the cells were viable and growing well at 200nM SN38 and at 10nM ixazomib (MLN9708). However, cell growth was completely inhibited when the same drug concentration was combined. 
A.
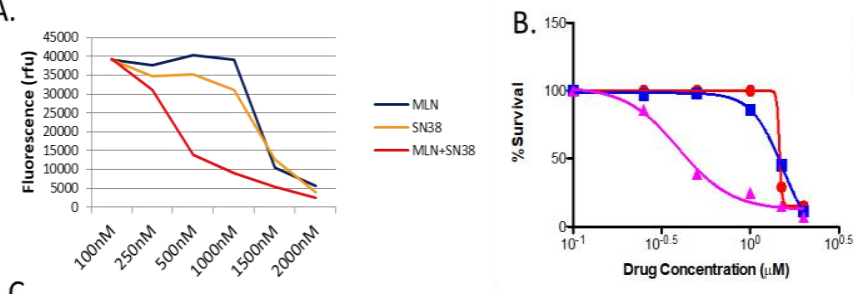

- MLN (IC50-1.464MM)

Fig. 4. Proteasome Inhibitor enhances CPT sensitivity: A. Cells were seeded on 96-well plates and treated with MLN9708/ixazomib, SN38 and MLN9708+SN38. After 24 hours the drugs were removed and cell viability assay was performed after 72 hours of drug treatment using Alamar Blue. B. Alamar blue assays were performed in triplicates and the IC50 values were determined by nonlinear regression of the dose-response data using Prism 5.0 for Mac OSX (GraphPad)
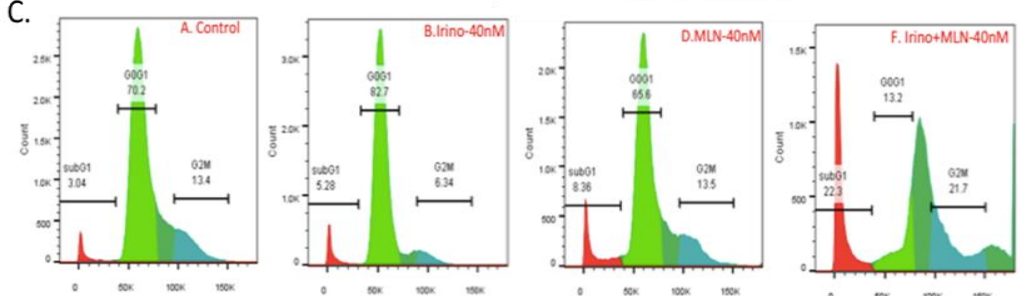

D.

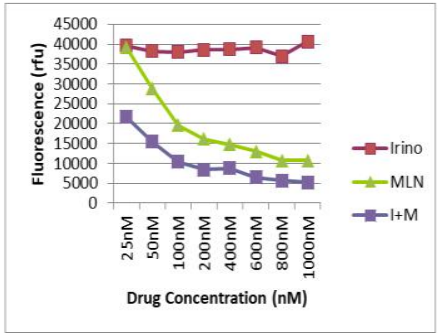

E.

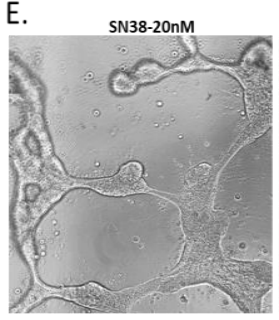
Software. C. HCT15 cells were treated with DMSO (control), SN38 (irinotecan), MLN9708 and combination of MLN9708 and SN38 for 24 hours, drugs were removed and cells were harvested after 72 hours of the drug treatment. Cells were fixed in $70 \%$ ethanol and DNA was stained with propidium iodide. Sub G1 assay was performed using LASRII. D. HCC 70 cells were treated with different concentrations SN38, MLN9708 and combination of SN38 and MLN9708 and cell viability assays were performed after 72 hours of the drug treatment. E. HCC 70 cells were grown in Matrigel and treated with different concentrations of SN38, MLN9708 and combination of SN38 and MLN9708 and after 72 hours the cells were visualized by bright field imaging.
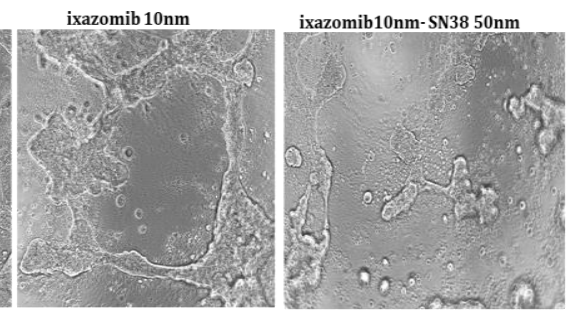

Future Directions: TopoI inhibitors represent one of the most extensively used chemotherapeutic agents. More recently, topoI inhibitors were successfully used as payloads in antibody drug conjugates (ADCs). ADCs are one of the fastest growing classes of cancer drugs and third generation of ADCs have shown significant improvements in achieving higher therapeutic index. There are three components in an ADC: cell surface protein binding antibody, a cytotoxic warhead, and a linker that conjugates the two. Humanized cell surface antibodies, like Herceptin, are extensively used in clinical oncology and linker chemistry has matured. However, effectively using cytotoxic agents, as warheads/ payloads in ADCs have remained challenging. In the last twenty years, nine ADCs have been FDA approved; however, only five cytotoxic payloads were used successfully. Out of the five approved payloads, two are topoI inhibitors. To increase the therapeutic index of ADCs, improvements have to be made either in potency of the cytotoxic agent to lower the minimum effective dose or in tumor selectivity to increase the maximum tolerated dose. Our studies on the resistance mechanisms of topoI 
inhibitors will be very helpful to improve the therapeutic index of ADCs with topoI inhibitors as payload.

Conclusions: Previously four different labs have demonstrated that; i) CPT induces topoI degradation in patient leucocytes and cancer cell lines; ii) topoI degradation is mediated by ubiquitin proteasomal pathway; iii) rate of topoI degradation varies in different cell lines; iv) the cells that degrade topoI rapidly are resistant to CPT. More recently, the pathway of proteasomal degradation of topoI was defined and the molecular determinants were identified. It was shown that deregulated kinase cascade ensures activated DNA-PKcs, a higher basal level of topoI-pS10 and CPT-induced rapid degradation of topoI. Notably, BRCA1 was identified as E3 ligase for CPT induced topoI ubiquitination and BRCA1 deficient cells were sensitive to CPT. We asked if CPT sensitivity can be enhanced by proteasomal degradation. The data presented here clearly indicates that proteasome inhibition reduces CPT induced proteasomal degradation of topoI and enhances CPT sensitivity significantly. This further validates the developed UPP pathway and opens up the possibility of a novel combination therapy. Furthermore, our understanding of CPT resistance will be very helpful in developing novel ADCs with topoI inhibitors as payload.

\section{References}

1. Sulistio, Y.A. and K. Heese, The Ubiquitin-Proteasome System and Molecular Chaperone Deregulation in Alzheimer's Disease. Mol Neurobiol, 2016. 53(2): p. 905-931.

2. Mansour, M.A., Ubiquitination: Friend and foe in cancer. Int J Biochem Cell Biol, 2018. 101: p. 80-93.

3. Hasselgren, P.O. and J.E. Fischer, The ubiquitin-proteasome pathway: review of a novel intracellular mechanism of muscle protein breakdown during sepsis and other catabolic conditions. Ann Surg, 1997. 225(3): p. 307-16. 
4. Qi, J. and Z.A. Ronai, Dysregulation of ubiquitin ligases in cancer. Drug Resist Updat, 2015. 23: p. 1-11.

5. Manasanch, E.E. and R.Z. Orlowski, Proteasome inhibitors in cancer therapy. Nat Rev Clin Oncol, 2017. 14(7): p. 417-433.

6. Barretina J, Caponigro G, Stransky N, Venkatesan K, et.al: The Cancer Cell Line Encyclopedia enables predictive modelling of anticancer drug sensitivity.

Nature. 2012 Mar 28; 483(7391

7. Garnett MJ, Edelman EJ, Heidorn SJ, Greenman CD, Dastur A, et.al: Systematic identification of genomic markers of drug sensitivity in cancer cells.

Nature. 2012 Mar 28;483(7391):570-5

8. Wang JC: Cellular roles of DNA topoisomerases: a molecular perspective. Nat Rev Mol Cell Biol 3:430-40, 2002

9. Hsiang, Y.H. and L.F. Liu, Identification of mammalian DNA topoisomerase I as an intracellular target of the anticancer drug camptothecin. Cancer Res, 1988. 48(7): p. 1722-6.

10. Hsiang YH, Wu HY, Liu LF: Topoisomerases: novel therapeutic targets in cancer chemotherapy. Biochem Pharmacol 37:1801-2, 1988

11. Liu LF, Desai SD, Li TK, et al: Mechanism of action of camptothecin. Ann N Y Acad Sci 922:1-10, 2000

12. Pommier Y: Topoisomerase I inhibitors: camptothecins and beyond. Nat Rev Cancer 6:789-802, 2006

13. Van Cutsem E, Kohne $\mathrm{CH}$, Hitre E, et al: Cetuximab and chemotherapy as initial treatment for metastatic colorectal cancer. N Engl J Med 360:1408-17, 2009

14. Heidelberger $C$, Chaudhuri NK, Danneberg $P$, et al: Fluorinated pyrimidines, a new class of tumour-inhibitory compounds. Nature 179:663-6, 1957

15. Patel JN, Fong MK, Jagosky M: Colorectal Cancer Biomarkers in the Era of Personalized Medicine. J Pers Med 9, 2019

16. Van Cutsem E, Huijberts S, Grothey A, et al: Binimetinib, Encorafenib, and Cetuximab Triplet Therapy for Patients With BRAF V600E-Mutant Metastatic Colorectal Cancer: Safety Lead-In Results From the Phase III BEACON Colorectal Cancer Study. J Clin Oncol 37:1460-1469, 2019

17. Elez E, Argiles G, Tabernero J: First-Line Treatment of Metastatic Colorectal Cancer: Interpreting FIRE-3, PEAK, and CALGB/SWOG 80405. Curr Treat Options Oncol 16:52, 2015

18. Matsuoka T, Yashiro M: Biomarkers of gastric cancer: Current topics and future perspective. World J Gastroenterol 24:2818-2832, 2018 
19. Nishida T: Treatment of gastric cancer in Asia: the missing link. Lancet Oncol 10:1027-8, 2009

20. Al-Batran SE, Homann N, Pauligk C, et al: Perioperative chemotherapy with fluorouracil plus leucovorin, oxaliplatin, and docetaxel versus fluorouracil or capecitabine plus cisplatin and epirubicin for locally advanced, resectable gastric or gastro-oesophageal junction adenocarcinoma (FLOT4): a randomised, phase 2/3 trial. Lancet 393:1948-1957, 2019

21. Wagner AD, Syn NL, Moehler M, et al: Chemotherapy for advanced gastric cancer. Cochrane Database Syst Rev 8:CD004064, 2017

22. Scherf, U., et al., A gene expression database for the molecular pharmacology of cancer. Nat Genet, 2000. 24(3): p. 236-44.

23. Szakács, G., et al., Predicting drug sensitivity and resistance: profiling $A B C$ transporter genes in cancer cells. Cancer Cell, 2004. 6(2): p. 129-37.

24. Tamura, H., C. Kohchi, R. Yamada, T. Ikeda, O. Koiwai, E. Patterson, J.D. Keene, K. Okada, E. Kjeldsen, K. Nishikawa, and et al., Molecular cloning of a cDNA of a camptothecin-resistant human DNA topoisomerase I and identification of mutation sites. Nucleic acids research, 1991. 19(1): p. 69-75.

25. Kubota, N., F. Kanzawa, K. Nishio, Y. Takeda, T. Ohmori, Y. Fujiwara, Y. Terashima, and N. Saijo, Detection of topoisomerase I gene point mutation in CPT-11 resistant lung cancer cell line. Biochemical and biophysical research communications, 1992. 188(2): p. 571-7.

26. Benedetti, P., P. Fiorani, L. Capuani, and J.C. Wang, Camptothecin resistance from a single mutation changing glycine 363 of human DNA topoisomerase I to cysteine. Cancer research, 1993. 53(18): p. 4343-8.

27. Rubin, E., P. Pantazis, A. Bharti, D. Toppmeyer, B. Giovanella, and D. Kufe, Identification of a mutant human topoisomerase I with intact catalytic activity and resistance to 9-nitro-camptothecin. The Journal of biological chemistry, 1994. 269(4): p. 2433-9.

28. Fujimori, A., W.G. Harker, G. Kohlhagen, Y. Hoki, and Y. Pommier, Mutation at the catalytic site of topoisomerase I in CEM/C2, a human leukemia cell line resistant to camptothecin. Cancer research, 1995. 55(6): p. 1339-46.

29. Chang, J.Y., J.F. Liu, S.H. Juang, T.W. Liu, and L.T. Chen, Novel mutation of topoisomerase I in rendering cells resistant to camptothecin. Cancer research, 2002. 62(13): p. 3716-21.

30. Ohashi, N., Y. Fujiwara, N. Yamaoka, O. Katoh, Y. Satow, and M. Yamakido, No alteration in DNA topoisomerase I gene related to CPT-11 resistance in human lung cancer. Japanese journal of cancer research : Gann, 1996. 87(12): p. 1280-7. 
31. Takatani, H., M. Oka, M. Fukuda, F. Narasaki, R. Nakano, K. Ikeda, K. Terashi, A. Kinoshita, H. Soda, T. Kanda, E. Schneider, and S. Kohno, Gene mutation analysis and quantitation of DNA topoisomerase I in previously untreated non-small cell lung carcinomas. Japanese journal of cancer research : Gann, 1997. 88(2): p. 160-5.

32. Tsurutani, J., T. Nitta, T. Hirashima, T. Komiya, H. Uejima, H. Tada, N. Syunichi, A. Tohda, M. Fukuoka, and K. Nakagawa, Point mutations in the topoisomerase I gene in patients with non-small cell lung cancer treated with irinotecan. Lung cancer, 2002. 35(3): p. 299-304.

33. Moisan, F., M. Longy, J. Robert, and V. Le Morvan, Identification of gene polymorphisms of human DNA topoisomerase I in the National Cancer Institute panel of human tumour cell lines. British journal of cancer, 2006. 95(7): p. 906-13.

34. Sirikantaramas, S., M. Yamazaki, and K. Saito, Mutations in topoisomerase I as a selfresistance mechanism coevolved with the production of the anticancer alkaloid camptothecin in plants. Proceedings of the National Academy of Sciences of the United States of America, 2008. 105(18): p. 6782-6.

35. Rubin E, Wood V, Bharti A, Trites D, Lynch C, Hurwitz S, Bartel S, Levy S, Rosowsky A,

Toppmeyer D and Kufe D. A phase I pharmacokinetic study of a new camptothecin derivative, 9-Aminocamptothecin. Clinical Cancer Research, 1995. 1:p.269-276

36. Beidler DR, Cheng YC. Camptothecin induction of a time- and concentrationdependent decrease of topoisomerase I and its implication in camptothecin activity. Molecular pharmacology 1995; 47: 907-914.

37. Desai SD, Liu LF, Vazquez-Abad D, D'Arpa P. Ubiquitin-dependent destruction of topoisomerase I is stimulated by the antitumor drug camptothecin. The Journal of biological chemistry 1997; 272: 24159-24164.

38. Desai, S.D., et al., Ubiquitin/26S proteasome-mediated degradation of topoisomerase I as a resistance mechanism to camptothecin in tumor cells. Cancer Res, 2001. 61(15): p. 5926-32.

39. Ando K, Shah AK, Sachdev V, et al: Camptothecin resistance is determined by the regulation of topoisomerase I degradation mediated by ubiquitin proteasome pathway. Oncotarget 8:43733-43751, 2017

40. Ando K, Tohme YH, Srinivasiah A, et al: Developing a Phosphospecific IHC Assay as a Predictive Biomarker for Topoisomerase I Inhibitors. J Histochem Cytochem 66:549-561, 2018

41. Ando K, Ozonoff A, Lee S, Voisine M, Parker J, Nakanishi R, Nishimura S, Yang J, Grace Z, Tran B, Diefenbach T, Maehara Y, Yasui H, Irino T, Salgia R, Terashima M, Gibbs P, Ramanathan R, Oki E, Mori M, Kulke M, Hartshorn K, and Bharti A Multi-Cohort Retrospective Validation of a Predictive Biomarker for Topoisomerase I Inhibitors. (Clinical Colorectal Cancer: In Press) 
42. Venkitaraman AR. Cancer susceptibility and the functions of BRCA1 and BRCA2. Cell 2002;108(2):171-82.

43. Wang Y, Cortez D, Yazdi P, Neff N, Elledge SJ, Qin J. BASC, a super complex of BRCA1-associated proteins involved in the recognition and repair of aberrant DNA structures. Genes \& development 2000;14(8):927-39.

44. Reid LJ, Shakya R, Modi AP, Lokshin M, Cheng JT, Jasin M, et al. E3 ligase activity of BRCA1 is not essential for mammalian cell viability or homologydirected repair of double-strand DNA breaks. Proceedings of the National Academy of Sciences of the United States of America 2008;105(52):20876-81 doi 10.1073/pnas.0811203106.

45. Sato K, Sundaramoorthy E, Rajendra E, Hattori H, Jeyasekharan AD, Ayoub N, et al. A DNA-damage selective role for BRCA1 E3 ligase in claspin ubiquitylation, CHK1 activation, and DNA repair. Current biology : CB 2012;22(18):1659-66 doi 10.1016/j.cub.2012.07.034.

46. Goldwasser F, Bae I, Valenti M, Torres K, Pommier Y. Topoisomerase I-related parameters and camptothecin activity in the colon carcinoma cell lines from the National Cancer Institute anticancer screen. Cancer research. 1995; 55(10):2116-21. 